\title{
La béance entre les scènes
}

\author{
Louise Dupré
}

\section{The Yawning (Gap) Between the Scenes}

Writing in the feminine in 1989 is to write against the monotonous senselessness of 'the normal' in order to give a form to my desire, a woman form, since form can't be neuter. Writing is a place of passage where I shed my conventional (normal) uniforms in order to think a new fiction which will give me back my voice in all its polytonality, all its bodily shock points. I write against myself, against the narrativity indoctrinated in me from infancy, memory as the anecdote of my fouryear old self who hadn't been subdued yet. But I also write memory as a zone of exploration where, through a reinvented fiction, I come to understand reality other-wise. In Bonheur (Happiness), I wanted to explore that kitsch word, how it is inseparable from suffering. My writing is made of the clashing of concepts, of literary genres, the transformation of story into a space where sense is confused with the sensual.I seek to knock out binary logic, to produce what might be called a logic of aporia, women-logic. I can't accept the destruction of the western subject of discourse without proposing a possible reconstruction where I can speak and pass from the object of masculine desire to the function of subject acting on myown desire. For a woman it's not a question of to be or not to be but rather how to be while not being.

Je pourrais dire $j^{\prime}$ écris contre la mémoire. Je pourrais tout aussi bien dire j'écris la mémoire, une mémoire blanche qui se refuse à mon bon souvenir. Ecrire au féminin en 1989, c' est contrecarrer le présent quand il faufile ses matières usées, les journées monotones où il ne se passe rien, où il ne peut rien se passer parce quel' intensitése refuse, les gens trop pressés enfermés dans leur bulle, quelques politesses comme bonjour comment vas-tu? auxquelles on répond très bien et toi sans prendre le temps des'arrêter. Le quotidien quise noie dans ses propres lieux communs. On penseà la fatalité, la vie passe, le temps, il 
s'agit de s'y faire. Serais-tu mésadaptée ma pauvre vieille, anormale, insatisfaite, pire folle parfois quand tout à coup il te semblequerienn'a de sens, qu'il te faut donner une forme à ton désir.

Quand je pense au mot forme, il me vient en tête le mot femme.Je ne peux imaginer de forme qui soit neutre. Qui, dans sa neutralité, me vide de mon bouillonnement intérieur: tendresse, inquiétude, colère, excès, passion. L'écriture, $c^{\prime}$ est pourmoi un lieu de passage où jequitte un à un tous mes uniformes: l'individue de classe moyenne, blanche, bien éduquée, modérée, sachant gardersa place, qui vote tous les quatre ans, au fédéral, au provincial, au municipal, parcourt le Devoir et l'Actualité, regarde à la télévision le Point, Actuel, Apostrophe, voit le dernier film à la mode.

Dire le mot femme, $c$ 'est pour moi penser une fiction nouvelle qui me permette de m'approcher au plus près de ma nudité, $d$ 'une pensée du corps qui à chaque fois me redonne ma voix, voix de tête, voix de gorge et de ventre, tour à tour chantante, éraillée ou explosive, toujours mienne cependant dans sa polytonalité, la tête, le ventre, la gorge comme autant de points chauds où se crée le texte.

Points chauds. Points de tension, de choc. J'ai dit bouillonnement intérieur. L'écriture, ça n'advient que dans la violence, à la limite de l'insoutenable. L'individue de bonne famille, blanche, bien éduquée ne se laisse pas si facilement dépouiller d'une conformité qu'elle a mis tant de temps à acquérir. Elle se rebelle, refuse de lâcher prise. J'écris contre moi-même, contre la narrativité qu'on m'a inculquée depuis la première enfance. J'écris contre la mémoire, si ce qu'on entend par le mot mémoire, c'est le souvenir pour le souvenir, l'anecdote, la fois où, à quatreans, tu avais dit à la voisine que tu refusais det' occuper deson petit dernier, la grand-mère qui avait murmuré à ta mère quelle tête forte, celle-là, pour une fille!

Les petits faits du passé encombrent le cerveau tant qu'on ne les transforme pas en une expérience véritable prenant sens dans une histoire des femmes encoreà retracer dans ses forces vives. Ça se dit alors autrement: à quatre ans, tu refusais ce qu'on attendait de toi. A quatre ans, on n'avait pas encore réussi à te mater. Ça viendrait beaucoup plus tard, à l'adolescence, quand tu comprendrais combien il était difficile pourunefille d'êtreen dehors des normes, combien les femmes le payaient cher: le rejet, la solitude, les maisons psychiatriques, parfois la mort, une douleur sans nom que tu te sentais incapable d'affronter seule. 
J'écris le mémoire si ce qu'on entend par ce mot, c'est un travail qui rattache le je au nous, établit des rapports inédits, zone d'exploration où, par une fiction sans cesse à réinventer, je comprends autrement la réalité, $j$ 'arrive à donner forme à un futur possible. Dans Bonheur par exemple, j'ai voulu explorer le mot bonheur, un mot usé jusqu'à la corde, un mot kitsch, exploité dans les chansonnettes populaires où amour rime avec toujours et corps avec encore. Un mot délaissé par une modernité québécoise qui, dans son effort pour penser autrement le sujet de discours, en est arrivée à proposer une vision de l'éclatement, de la destruction extrême. Comme femme, comme être qui n'a pas été socialement reconnu comme sujet, je ne peuxaccepter l'éclatement pour l'éclatement, parce que c'est ce que j'ai toujours connu: le morcellement, la schize. Comme femme, je ne peux accepter la destruction du sujet occidental du discours sans proposer une possible reconstruction où je puisse trouver une place qui me convienne pour prendre la parole.

Dans Bonheur, j'ai fait le pari suivant: dire oui le bonheur peut exister, oui le bonheur est possible si on cesse de le voir comme séparé dela souffrance, de la mélancholie. Quand j'ai écrit: 'Le bonheur n'est pas l'envers de la mélancholie mais son mur de lumière', voilà précisément ce à quoi j'ai voulu faire référence. Arrêter de voir le je comme un bloc homogène, les états émotifs tout en noir ou tout en blanc. L'esprit coupé de la matière, l'intellect coupé du corps, la culture opposée à la nature, n'est-ce pas la façon de voir la réalité à laquelle la culture occidentale nous a habituées, les hommes étant du côté du rationnel et les femmes, du côté du corporel?

Comme écrivaine de fiction, je veux précisément travailler à réunir ce qui a été disjoint, penser ensemble les contraires, mettre les antonymes du dictionnaire en contact les uns avec les autres. Comment expliquer? Mon écriture se trame à partir de certains mots qui s'imposent, le mot chambre, les mots demande, amour, puis enfance, mort, noir, catastrophe, des mots incontournables avec lesquels il faut composer, des mots qui insistent même si j'essaie de les éviter. Ça attire, des centres de gravité, et mon énergie passe à leur faire trouver leur forme sans entrer totalement dans leur orbite. Eprouver la peur d'aller trop loin. Ne plus pouvoir revenir,être enfermée dans ces mots, mort, noir, catastrophe. Il faut chercher un équilibre. Ecrire est une épreuve de force. Il y a des mots qui peuvent détruire.

A la table de travail, il y a cette partie de moi qui se laisse subjuguer 
par les mots qui m'ont faite ce quejesuis, mais il y a aussi cette partiede moi qui essaie de les tenir à distance et qui, pour ce faire, a besoin des mots demain, rêve, immensité, bonheur. Ecrire, c'est pour moi entrer dans la contradiction, lui donner ancrage. Mon écriture se fait dans le choc: choc des concepts, mais aussi choc des genres littéraires. Je ne peux penser la poésie sans la narrativité et la narrativité sans une théorie du féminin. En cesens, la poésie en prose me permet d'inventer des formes, jamais les mêmes, jamais figées, qui fassent coïncider le dire de ma parole avec un faire toujours à renouveler.

Bref, je résiste constamment aux étiquettes des genres littéraires, je cherche à exercer une vigilance qui me fasse déroger aux beaux modèles, traditionnels ou modernes, pour que résonne ma propre voix. La voix, la musique vocale, est précisément ce qui arrache les signes à la transparence d'une histoire soumise à l'impersonnalité d'un narrateur-dieu, elle travaille dans la subjectivité pour régresser jusqu'aux masses informes d'une mémoire toujours fugitive. Une mémoire qu'on ne saura jamais rattraper. Une présence qui devient absence à mesure que se noircit la page.

Ecrire, voilà. J'écoute les mots se raconter, pour m'éloigner des scénarios prévisibles. Pour que la fiction se creuse dans ce manque même. A chaque fois que je commence un texte poétique en prose, j'ignore ce que je vais découvrir: je sais seulement que je trouve l'envers des histoires. Là où le rêve rencontre l'utopie d'une langue épurée des contes de l'enfance. Symphonie de formes et de couleurs qui s'imposent comme autant d'images déçues.

L'utopie del'écriture, $c$ 'est à fleur de peau ce quelenarrateur-dieu a laissé en suspens. Il était une fois ... la béance entre les scènes, le beau désordre, la transformation du récit en un espace où la signification cède à l'émotion: mouvement et énergie, confusion du sens avec le sensuel. Je chercheà bousculer la logiquebinaire, à produiredans mon écriture ce qu' on appelle une logique de l'aporie, une logique-femme qui trouve sa figure dans l'oxymoron. Quand à la fin de Bonheur j'ai écrit: 'Je ne suis pas la femme que je suis', j'ai voulu exprimer par cette formule l'état contradictoire auquel les mots me confrontent.

La confrontation dont il est question ici, c'est le rapatriement, chez le sujet, des fonctions appartenant à des actants qui s'opposent dans l'espace du récit populaire traditionnel: le sujet versus l'opposant, pour reprendre la terminologie de A.J. Greimas. On pourra me rétorquer que depuis la phrase 'je est un autre' de Rimbaud, cela est admis. 
Je répondrai simplement que, pour la femme, il s'agit d'une situation plus ambiguë encore puisqu'il s'agit justement de devenir sujet, c'est-à-dire de passer d'objet du désir masculin à la fonction de sujet pour s'assumer comme actante de son propre désir. En d'autres termes, il nes'agit pas, lorsqu' on est une femme, de se demander comment être ou ne pas être, il faut plutôt apprendre comment être tout en n'étant pas, parvenirà s'assumer positivement dans la contradiction.

Cette démarche requiert un double mouvement: non seulement la recherche de figures stylistiques qui puissent mettre en scène le je-femme, mais aussi l'invention d'images pouvant rendre compte de la forme d'un corps féminin quise tient toujours un peu en dehors de la réalité pour susciter l'imagination, le rêve, le risque d'une fiction débarrassée des stéréotypes.

Dérangée et dérangeante, ma propre fiction, voilà comment je la vois, puisqu' elle remet en cause tout aussi bien les formes de la poésie que celles du récit. Je lui prête une allure de femme aux multiples visages, mouvants, changeants, une allure qui transfigure où je reconnaisse mes propres traits parce que les façons traditionnelles de réciter ne sonnent pas juste. Je ne veux plus entendre les autres raconter mon histoire. Je veux en assumer moi-même la narration. A quatre ans, jele voulais déjà.

J'écris contre la mémoire grise. Inventer une mémoire qui projette de l'audace sur la conformité. 